\title{
Postural Stability and Academic Achievements among Fifth Graders: An Experimental Field Study
}

\author{
Miri Shachaf ${ }^{1}$, Roni Laslo-Roth ${ }^{2}$ and Eyal Rosenstreich ${ }^{2 *}$ \\ ${ }^{1}$ Department of Physical Education, Givat Washington Academic College of Education, Israel \\ ${ }^{2}$ Department of Behavioral Sciences, Peres Academic Center, Israel
}

\begin{abstract}
Postural stability was previously associated with academic achievements among primary school children, but only little is known on how cognitive-motor processes underlie academic achievements. This research was primarily aimed at investigating cognitive-motor performance (e.g., single vs. dual-task gait) and it correlates with achievements in math and language-arts. Fifth-graders $(N=86)$ performed several motor tasks while their mediolateral trunk sway was assessed using a Kinect 3D sensor. Achievements in math and language-arts were assess one's comply with motor task demands using standardized tests. Results showed that motor tasks predicted achievements in language-arts but not in math. More importantly, employing stability perform anceratios revealed that the ability to maintain static-balance in the absence of visual-information (standing on dominant foot, blindfolded/standing on dominant foot, eyes opened) as well as the ability to maintain dynamic-balance while carrying bean bag over the head (tandem walk with a bean bag on top of the head/tandem walk) were sole predictors of language-arts. This study stresses the importance of examining children in their natural environment and of employing performance ratio-scores to better understand the cognitivemotor processes underlying academic-performance.
\end{abstract}

\section{Keywords}

Static balance, Dynamic balance, Academic achievements, Kinect, Attention, Inhibitory processes

\section{Introduction}

Academic achievements are contributed by various social and cognitive factors, all directly or indirectly linked to executive functions (EF). EF is an umbrella term which refers to one's ability to plan, execute, or withhold actions, and to manage cognitive resources (e.g., to allocate attentional resources to task or tasks) [1]. EF were shown to be positively correlated with academic achievements in language arts and math, such that stronger executive abilities typically manifested with higher academic scores during elementary and middle school [2].

EF also entails the control over movement and some motor activities. Specifically, gait and balance performance is mainly determined by one's own ability to initiate or maintain motor action (i.e., planning and executing), to adjust or withhold an action (i.e., response regulation and inhibition), and by the ability to divide attentional resources and allocate them to different simulations actions (i.e., divided attention) [3]. This intimate connection between EF and motor activity led scholars to suggest over the past few years, that motor activities may benefit academic achievements by strengthening EF [4].

Indeed, some cross-sectional studies showed academic achievements to be positively associated with cardio- respiratory fitness among elementary school students (e.g., Castelli, Hillman, Buck, \& Erwin [5]). Similarly, in a longitudinal study in which data were collected from preschool through the 5th grade, it was found that parents' evaluation of their child's physical activity was positively related with academic achievements in both mathematics and reading skills [6]. Furthermore, other studies which examined the effects of physical activity on the academic achievements among $6^{\text {th }}$ grade and middle-school students, showed that students who engaged in some vigorous activity scored higher in standardized and non-standardized tests in reading and math, compared to students who reported no vigorous activity ([79]; for similar effect among high-school female students, see Shachaf, Katz, \& Shoval [10]).

*Corresponding author: Eyal Rosenstreich, Department of Behavioral Sciences, Peres Academic Center, 10 Peres St., Rehovot, Israel

Accepted: May 07, 2019

Published online: May 09, 2019

Citation: Shachaf M, Laslo-Roth R, Rosenstreich E (2019)

Postural Stability and Academic Achievements among Fifth Graders: An Experimental Field Study. Ann Cogn Sci 3(1):78-85 
So far, we have used the commonly used terms "physical activity" and "motor performance" in order to refer to motor task demands. Nonetheless, these terms are too broad and vague, and may represent a wide range of measures. In this study, we define motor performance in terms of maintaining balance, which is the process of maintaining the body's center of gravity vertically over the base of support. Maintaining balance is based on a rapid, continuous feedback from visual, vestibular and somatosensory structures and as a result performs smooth and coordinated neuromuscular actions [11]. Balance is typically divided into two main categories: Static and dynamic. Static balance defined as the ability to maintain a base of support with minimal movement; incontrast, dynamic balance is conceived as the ability to perform a task while maintaining or regaining a stable position [12], or as the ability to maintain or recoup balance on an unstable surface with minimal extraneous motion [13].

Balance skills were previously associated with various cognitive abilities among boys aged 5-6 years [14], reading and math skills among children aged 7-11 years [15], spatial skills among 10-years-old [16], and executive functioning in adolescents [17]. Similarly, a longitudinal study on 126 children showed that balance skills at kindergarten explained a significant amount of the variance in proportional reasoning skills at the end of first grade [18].

A variety of methods were employed in the literature to assess static and dynamic balance performance. In the school or sport literature, it is common to assess balance using timed performance. For example, Frick and Möhring [18]. defined children's static balance ability as the duration in seconds they were able to stand on one foot. However, whereas timed performance may be easily assessed, it may not reflect the quality of one's motor performance [19]. In particular, Weiss, et al. [19] showed that dynamic balance may be more accurately assessed through trunk sway variability rather than overall performance time. Indeed, in the movement literature, it is common to assess medio-lateral (ML) trunk sway variability as a measure of balance quality.

Nonetheless, ML sway may not be easily assessed in field studies, due to the need for participants to carry sensitive measuring devices (e.g., an accelerometer). However, some studies suggested that a Microsoft Kinect remote 3D sensor may be reliable, valid, and efficient instrument to assess balance in and out of the lab $[20,21]$. This instrument lies in the heart of the present study.

The purpose of the present study was to further investigate the associations between balance and academic achievements among $5^{\text {th }}$ graders. The study was guided by two major goals. First, we sought to generalize the current knowledge regarding balance and academic achievements to the objective quality of performance, assessed by trunk sway. To this end, instead of time-based motor assessment, dynamic and static balance were assessed using a Kinect sensor which remotely captured children's ML sway. Kinect-based assessment enables the examination of postural stability in close-to-natural settings; that is, participants are not required to wear or be in contact with the motion sensor and are free to move and act as they typically do [22].
The second goal of the present study was to indirectly assess attentional and inhibitory processes through dynamic and static balance performance. Specifically, it is common to manipulate attentional demands by performing the primary task alone (i.e., a single task condition) or simultaneously with another task (i.e., a dual-task condition); similarly, it is common to manipulate the availability of visual information during motor task by performing the task with eyes open and again with eyes closed. These conditions may be employed for the extraction of performance scores (e.g., effect size) that may be indicative of certain cognitive states. For example, it was recently demonstrated that a performance score reflecting the availability of visual information during static balance task (i.e., a measure derived from performance with eyes open in relation to blindfolded), was predicted by participants' ability to be interoceptively aware [22]. Put differently, in the present study we sought to assess not only raw motor performance under common motor tasks, but also performance ratio scores which are commonly assumed to reflect attentional cost, availability of visual information, and inhibitory motor control.

Academic achievements were examined in three language arts domains and three mathematics domains. In line with Frick \& Mohring [18], it was hypothesized that stability during static balance tasks will predict achievements in math, such that lower $\mathrm{ML}$ variability will be accompanied by higher math scores. Furthermore, achievements in language arts were hypothesized to be predicted by both dynamic and static balance performance, such that lower ML variability will be accompanied by better performance in language arts test scores.

\section{Method}

\section{Participants}

Overall, $865^{\text {th }}$ graders (aged $10-13$ years, $M=10.76, S D$ $=0.40 ; 53.5 \%$ females) participated in the research. All the participants were sampled at an elementary school located in a large city at the center of Israel. Information regarding children's Socio-Economic Status (SES) was not collected, but school records indicated that house hold's socio-economic standing was largely middle class. All children were native Hebrew speakers with no visible or known motor deficiencies (except for one hypotonic student). In order to avoid social or affective implications, all children were allowed to participate in the study; however, 10 of these children were later excluded from analyses due to hypotonic $(n=1)$, being diagnosed with various cognitive deficiencies (e.g., language impairment; $n$ $=5), \mathrm{BMI}<13(n=2)$, or $\mathrm{BMI}>19(n=2)$. Verbal and written consent of participation were provided by the children and their parents, respectively, and the study was approved by the institutional ethics committee and by the school principal.

\section{Tasks and measures}

Motor tasks: Participants individually performed six tasks over approximately 3 (length) $\times 1.5$ (width) meters testing space; three dynamic balance tasks and three static balance tasks, as follows:

Static balance tasks (all carried on the floor for 21 seconds each) 
Citation: Shachaf M, Laslo-Roth R, Rosenstreich E (2019) Postural Stability and Academic Achievements among Fifth Graders: An Experimental Field Study. Ann Cogn Sci 3(1):78-85

(1) Standing on dominant foot, eyes open.

(2) Standing on dominant foot, eyes open with a beanbag on top of the head.

(3) Standing on dominant foot, blindfolded.

Dynamic balance tasks (all were self-paced and carried on a beam of a reverse Swedish bench $(\mathrm{L}: 285 \mathrm{~cm}, \mathrm{~W}: 9 \mathrm{~cm}, \mathrm{H}$ : $28 \mathrm{~cm})$.

(1) Tandem walk (Walking hill to toe along a straight line)

(2) Tandem walk with a beanbag on top of the head

(3) Dual tasking. The primary task was tandem walk, which was performed along with a secondary task of selective attention. The secondary task was d2 [23], in which participants were to find and cross with two lines the letter " $d$ " in a randomly mixed set consisting of "b", "d", "p", and "q". Set size was 40 letters, 10 letters of each type. Participants were instructed to perform both tasks equally, as best as they can. The task-page was placed on a clipboard held by the student.

Instruments: Motor performance was assessed using a Microsoft Kinect 3D sensor connected to a Windows 10 operated Core-i3 laptop. The Kinect sensor was primarily designed by Microsoft as a remote game controller, which captures users' fine and gross movements and translate them to onscreen actions. The Kinect's ability to identify body position in 3D-space was employed in order extract data regarding participants' ML trunk sway. Specifically, the Kinect SDK provided by Microsoft identifies body limbs position in 3D space. Participants' torso position was extracted from these raw data, and $\mathrm{ML}$ deviations from upright straight stance (in degrees) were sampled at a rate of $25 \mathrm{~Hz}$ such that higher deviation score represented greater sideways tilt of the torso relative to the upright stance. Split-half reliabilities were calculated by correlating between mean performances in the first and second halves of each task (that is, performance in the first and last 10.5 seconds or 262 data points) and was found high for all motor tasks (Spearman-Brown split-half corrections varied between 0.82 to 0.95 ).

$\mathrm{ML}$ data were processed to produce two types of scores: First, six raw stability scores which represented performance variability in each of the six motor tasks (i.e., high variability represented less stability); Second, four ratio scores were calculated to represent inhibitory control during gait, attentional cost during gait, inhibitory control during static balance, and the availability of visual information, as follows: Inhibitory control during gait was defined as the effect of beanbag during walking, and was calculated as the ratio between the stabilities in Dynamic Task 2 and Dynamic Task 1 (i.e., Ratio dynamic 2/1).Attentional cost during gait was defined as the effect of dual task during walking, and was calculated as the ratio between the stabilities in Dynamic Task 3 and Dynamic Task 1 (i.e., Ratio dynamic 3/1). Inhibitory control during static balance was defined as the effect of bean bag while standing on dominant foot, and was calculated as the ratio between the stabilities in Static Task 2 and Static Task 1 (i.e., Ratio static 2/1); and finally, the availability of visual information was defined as the effect of being blind folded while standing on dominant foot, and was calculated as the ratio between the stabilities in Static Task 3 and Static Task 1 (i.e., Ratio static 3/1). High ratio scores represented more variability in the inhibition of movement while carrying bean bag during dynamic balance, while maintaining dynamic balance in the lack of attention (i.e., high attentional cost), in the inhibition of movement while carrying beanbag during static balance, and while maintaining balance in the absence of visual information, respectively.

Academic achievements: Nationally standardized tests in mathematics and language arts. The language arts test consisted of three domains: A) Reading comprehension: Understanding the meaning of the text, interpreting, processing, and evaluating textual, structural, and linguistic components; B) Expression in writing: Content, structure, and use of language were assessed through a short essay; and C) Grammar: Proper use of linguistic forms and structures, linguistic awareness and linguistic knowledge. The math test consisted of three domains: A) Numbers and operations in simple fractions and decimals; B) Natural numbers and operations including 0 and data analysis; C) Geometry and measurements.

Demographics: Some demographic information was collected, as the date of birth, gender, and type and frequency of extra-curriculum physical activity.

\section{Procedure}

The study was conducted during school hours, that is, from 8:30 am to 1:30 pm over two days, at the school gym where the students study physical education. Before the students arrived to the gym, the experimenters set up the bench, the Kinect, and the computer. The Kinect was placed on a microphone stand fitted to firmly accommodate the sensor. The stand's height was adjusted to $100 \mathrm{~cm}$ and was placed about $10 \mathrm{~cm}$ in front of the end of the beam so it would capture participant's torso throughout the walking distance. The computer was placed on a small table behind the stand. Both computer and sensor positions were not altered throughout the entire study. Using a paper masking tape, the experimenters marked the position of the bench on the floor along with another position designated for the static balance tasks. The students entered the gym in groups of 6-8 and sat on scouts' chairs. The students first filled out the demographic questionnaire, and then were asked to practice finding the letter " $d$ " on a the $d 2$ practice sheet (which was similar to the actual test sheet but consisted of a smaller set size of 20 letters), while waiting for their turn to perform the motor tasks. The nature of the upcoming tasks was described by the experimenter, and participants were instructed to walk slowly during the dynamic tasks. Each student was individually tested and performed the six motor tasks in two blocks. In one block, the three dynamic tasks were carried, and in the second block, the static tasks were carried. The order of the tasks in each block was fixed as described above, but the presentation order of the blocks was counterbalanced across participants. When the static balance block was carried, the experimenters moved the beam aside and the participant performed the tasks in a premarked position located $200 \mathrm{~cm}$ from the sensor. When the dynamic balance block was carried, the experimenters placed 
Citation: Shachaf M, Laslo-Roth R, Rosenstreich E (2019) Postural Stability and Academic Achievements among Fifth Graders: An Experimental Field Study. Ann Cogn Sci 3(1):78-85

back to its pre-marked position.

\section{Statistical Analysis}

Sample size was determined based on a small-to-medium dual-task effect size (Cohen's $d=0.36 ; N=103$ ) obtained in previous study using the Kinect [23]. G*Power software [24] revealed that a sample size of 68 was needed in order to gain statistical power of $90 \%$. Several hierarchical multiple regressions were performed in order to examine the extent to which academic achievements were predicted by motor performance. Because background variables tend to explain some of the variance of academic achievements and motor performance, these variables were entered to the first block of each regression analysis. Furthermore, because the motor tasks employed in this study shared common characteristics, they were selected onto the second block of each regression analysis using stepwise.

\section{Results}

\section{Preliminary data treatment}

Motor performance data was first processed in order to refine the assessment of stability. Specifically, for each participant and in each task, outliers were excluded from analysis (we followed the common cutoff of \pm 2 SDs from mean performance; Osler, Tersteeg, Reynolds, \& Loram, [25]). Then, ML variability was assessed as the standard deviation score of the performance in each task, such that high score represented less stable performance. This yielded six stability scores corresponding with the six motor tasks assessed in this study. These stability scores were employed for the calculation of the four performance ratios. Descriptive statistics are presented in Table 1.

Next, several hierarchical regressions were conducted in order to examine the unique contribution of each motor task to academic achievements when background variables were controlled. In particular, we controlled for sex, age, dominant foot, the extant of engagement with extra-curriculum physical activity, the order of tasks carried in the study, and for learning disabilities ( $L D, n=5$ ). To this end, six hierarchical regression analyses were conducted each with one of the six academic scores as the predicted variable. In each analysis, the background variables were entered simultaneously into the first block; the six motor performance variables (three dynamic and three static), were entered into the second block using stepwise selection. This method was employed to reduce statistical errors due to the common motor bases underlying these tasks. Regression coefficients are presented in Table 2.

Table 2 reveals that academic achievements were predicted mainly by dynamic tasks, but these effects were limited to reading comprehension and total verbal scores. In particular, ML trunk variability during the simple gait

Table 1: Descriptive statistics of motor performance and academic achievements scores.

\begin{tabular}{|c|c|c|c|c|c|c|}
\hline & Mean & SD & Min. & Max. & Skewness & Skew SE \\
\hline \multicolumn{7}{|l|}{ Raw ML sway variability } \\
\hline Dynamic 1 & 4.44 & 2.67 & 1.22 & 17.18 & 2.50 & 0.28 \\
\hline Dynamic 2 & 2.90 & 2.15 & 0.90 & 17.82 & 2.85 & 0.28 \\
\hline Dynamic 3 & 6.89 & 3.34 & 2.55 & 14.91 & 0.65 & 0.28 \\
\hline Static 1 & 2.55 & 2.09 & 0.40 & 12.58 & 2.47 & 0.29 \\
\hline Static 2 & 1.64 & 0.97 & 0.48 & 5.38 & 1.47 & 0.29 \\
\hline Static 3 & 5.47 & 3.48 & 1.01 & 14.95 & 0.92 & 0.29 \\
\hline \multicolumn{7}{|l|}{ Motor performance ratios } \\
\hline Ratio Dyn. 2/1 & 0.73 & 0.36 & 0.08 & 1.73 & 0.88 & 0.28 \\
\hline Ratio Dyn. 3/1 & 1.87 & 1.18 & 0.24 & 6.01 & 1.57 & 0.28 \\
\hline Ratio Stat. 2/1 & 0.83 & 0.55 & 0.22 & 3.03 & 2.41 & 0.29 \\
\hline Ratio Stat. 3/1 & 2.99 & 2.53 & 0.39 & 12.03 & 1.71 & 0.29 \\
\hline \multicolumn{7}{|l|}{ Academic achievements } \\
\hline Grammar & 86.65 & 11.60 & 56 & 100 & -0.95 & 0.28 \\
\hline Written expression & 81.81 & 15.84 & 38 & 100 & -0.63 & 0.28 \\
\hline Reading comprehension & 83.34 & 12.41 & 45 & 100 & -1.22 & 0.28 \\
\hline Total Verbal & 83.54 & 10.93 & 53 & 98 & -0.98 & 0.28 \\
\hline Whole numbers & 81.19 & 17.96 & 23 & 100 & -1.11 & 0.28 \\
\hline Fractions & 75.61 & 24.87 & 6 & 100 & -1.19 & 0.28 \\
\hline Geometry & 86.31 & 17.29 & 25 & 100 & -1.56 & 0.28 \\
\hline Total Math & 79.73 & 18.79 & 24 & 100 & -1.30 & 0.28 \\
\hline
\end{tabular}

Note: ML: Medio-lateral; Dyn: Dynamic balance task; Stat: Static balance task. Raw ML scores represent the standard deviations of trunk tilt from body center (in degrees). 
Citation: Shachaf M, Laslo-Roth R, Rosenstreich E (2019) Postural Stability and Academic Achievements among Fifth Graders: An Experimental Field Study. Ann Cogn Sci 3(1):78-85

Table 2: Standardized regression coefficients of background and motor variables as predictors and academic achievements as the predicted variables.

\begin{tabular}{|c|c|c|c|c|c|c|c|c|}
\hline \multicolumn{9}{|c|}{ Predicted variables } \\
\hline \multirow[b]{2}{*}{ Predictors } & \multicolumn{4}{|c|}{ Language arts } & \multicolumn{4}{|c|}{ Mathematics } \\
\hline & Grammar & $\begin{array}{l}\text { Written } \\
\text { exp. }\end{array}$ & $\begin{array}{l}\text { Reading } \\
\text { comp. }\end{array}$ & Total & $\begin{array}{l}\text { Whole } \\
\text { numbers }\end{array}$ & Fractions & Geometry & Total \\
\hline \multicolumn{9}{|l|}{ Block 1 (enter) } \\
\hline Sex & -0.137 & $0.436^{* *}$ & -0.176 & -0.007 & 0.104 & -0.113 & -0.057 & -0.038 \\
\hline Age & -0.009 & -0.026 & -0.038 & -0.017 & -0.152 & -0.108 & -0.198 & -0.153 \\
\hline Dominant foot & -0.073 & -0.076 & -0.002 & -0.052 & -0.143 & $-0.229^{a}$ & $-0.236^{*}$ & -0.225 \\
\hline Physical activity & -0.216 & -0.153 & -0.071 & -0.126 & -0.087 & -0.142 & -0.167 & -0.143 \\
\hline Task order & 0.040 & -0.077 & 0.011 & -0.005 & -0.022 & -0.119 & 0.129 & -0.049 \\
\hline LD & -0.097 & $-0.231^{a}$ & $-0.252^{*}$ & $-0.286^{*}$ & -0.209 & $-0.279^{*}$ & $-0.311^{*}$ & $-0.297^{*}$ \\
\hline \multicolumn{9}{|c|}{ Block 2 (stepwise) } \\
\hline Dynamic 1 & & & $-0.569^{* *}$ & $-0.492^{* *}$ & & & & \\
\hline Dynamic 2 & & & $0.426^{* *}$ & $0.395^{* *}$ & & & & \\
\hline \multicolumn{9}{|l|}{ Dynamic 3} \\
\hline \multicolumn{9}{|l|}{ Static 1} \\
\hline \multicolumn{9}{|l|}{ Static 2} \\
\hline Static 3 & $-0.383^{* *}$ & & & & & & & \\
\hline Model $R^{2}$ & 0.22 & 0.34 & 0.31 & 0.32 & 0.11 & 0.18 & 0.22 & 0.18 \\
\hline Model $F$ & 2.36 & 5.07 & 3.16 & 3.26 & 1.16 & 2.12 & 2.78 & 2.11 \\
\hline Model $p$ & 0.035 & $>0.001$ & 0.005 & 0.004 & 0.342 & 0.064 & 0.019 & 0.065 \\
\hline
\end{tabular}

Note: LD: Learning disabilities; Written exp: Written expression; Reading comp: Reading comprehension; ML scores in Block 2 were assessed as the standard deviations of trunk tilt from body center (in degrees); ${ }^{*} p<0.05,{ }^{* *} p<0.01,{ }^{a} p<0.06$.

task (asassessed in Dynamic Task 1) negatively predicted achievements in reading comprehension and total verbal score, such that better gait stability (less variability) predicted better academic performance in these domains. In contrast, gait performance while carrying a bean bag (asassessed in Dynamic Task 2) positively predicted achievements in reading comprehension and total verbal scores, such that less stable gait performance (more variability) was accompanied by higher academic achievements in these domains. Finally, the ability to maintain balance in the absence of eyesight (asassessed in Static Task 3) negatively predicted grammar scores, such that better ability to maintain balance in the absence of eyesight was accompanied by higher grammar scores. No effect was observed for math.

Next, we repeated these analyses with the exception that the aforementioned motor tasks were now replaced with the four performance ratios as predictors. Regression coefficients are presented in Table 3.

Table 3 reveals that the ratio scores unfolded a different picture of the relationship between motor performance and academic achievements than provided by the raw stability scores. Specifically, achievements in grammar, as well as total language arts scores, were positively predicted by Ratio Dynamic 2/1, such that less body control during gait (less stability) predicted higher grammar scores; grammar was also negatively predicted by Ratio Static $3 / 1$, such that students who were more stable in the absence of visual information were better in grammar. Achievements in written expression were negatively predicted by Ratio Static $3 / 1$, indicating that better balance in the absence of eyesight was accompanied by higher achievements in this domain. Finally, Ratio Dynamic $2 / 1$ positively predicted achievements in reading comprehension, indicating that less body control during gait (less stability) predicted better comprehension. As before, no effects were observed for math.

\section{Discussion}

This study was aimed at investigating the associations between balance skills and academic achievements, and to extend existing knowledge by employing continuous assessment of motor performance. To this end, several static and dynamic balance tasks were assessed. As predicted, more stability in a single gait task predicted better academic achievements in comprehension and overall language arts scores. Similarly, as predicted, better ability to maintain balance while standing blindfolded on one foot predicted higher achievements in grammar. These findings support previous reports of an intimate connection between balance skills and academic achievements in language arts (e.g., Lopes, Santos, Pereira, \& Lopes; Son \& Meisels [26,27]), and further generalize this connection to ML trunk sway. However, in contrast with our predictions and not in-line with previous findings, the association between balance and academic 
Citation: Shachaf M, Laslo-Roth R, Rosenstreich E (2019) Postural Stability and Academic Achievements among Fifth Graders: An Experimental Field Study. Ann Cogn Sci 3(1):78-85

Table 3: Standardized regression coefficients of background variables and performance ratios as predictors and academic achievements as the predicted variables.

\begin{tabular}{|c|c|c|c|c|c|c|c|c|}
\hline \multicolumn{9}{|c|}{ Predicted variables } \\
\hline \multirow[b]{2}{*}{ Predictors } & \multicolumn{4}{|c|}{ Language arts } & \multicolumn{4}{|c|}{ Mathematics } \\
\hline & Grammar & $\begin{array}{l}\text { Written } \\
\text { exp. }\end{array}$ & $\begin{array}{l}\text { Reading } \\
\text { comp. }\end{array}$ & Total & $\begin{array}{l}\text { Whole } \\
\text { numbers }\end{array}$ & Fractions & Geometry & Total \\
\hline \multicolumn{9}{|l|}{ Block 1 (enter) } \\
\hline Sex & -0.006 & $0.552^{* *}$ & -0.088 & 0.155 & 0.104 & -0.113 & -0.057 & -0.038 \\
\hline Age & 0.001 & -0.047 & -0.112 & -0.096 & -0.152 & -0.108 & -0.198 & -0.153 \\
\hline Dominant foot & -0.002 & -0.013 & 0.018 & 0.026 & -0.143 & $-0.229^{a}$ & $-0.236^{*}$ & -0.225 \\
\hline Physical activity & -0.140 & -0.094 & -0.144 & -0.142 & -0.087 & -0.142 & -0.167 & -0.143 \\
\hline Task order & -0.044 & -0.099 & -0.064 & -0.096 & -0.022 & -0.119 & 0.129 & -0.049 \\
\hline LD & -0.090 & -0.194 & -0.190 & -0.196 & -0.209 & $-0.279^{*}$ & $-0.311^{*}$ & $-0.297^{*}$ \\
\hline \multicolumn{9}{|c|}{ Block 2 (stepwise) } \\
\hline Ratio Dyn. 2/1 & $0.256^{*}$ & & $0.312^{*}$ & $0.304^{*}$ & & & & \\
\hline \multicolumn{9}{|l|}{ Ratio Dyn. 3/1 } \\
\hline \multicolumn{9}{|l|}{ Ratio Stat. 2/1 } \\
\hline Ratio Stat. 3/1 & $-0.416^{* *}$ & $-0.352^{* *}$ & & $-0.296^{*}$ & & & & \\
\hline Model $R^{2}$ & 0.31 & 0.45 & 0.18 & 0.32 & 0.11 & 0.18 & 0.22 & 0.18 \\
\hline Model $F$ & 3.18 & 6.54 & 1.74 & 3.29 & 1.16 & 2.12 & 2.78 & 2.11 \\
\hline Model $p$ & 0.005 & $>0.001$ & 0.119 & 0.004 & 0.342 & 0.064 & 0.019 & 0.065 \\
\hline
\end{tabular}

Note: LD: Learning disabilities; Written exp: written expression; Reading comp: Reading comprehension; ${ }^{*} p<0.05 ;{ }^{* *} p<0.01 ;{ }^{a} p<0.06$.

achievements was limited only to language arts and was not observed for none of the three math domains examined in this study.

Two explanations may account for the differential associations between balance skills and achievements in language compared to math. First, this incompatibility with previous findings may be due to the measures employed. Specifically, it has been argued before that time-based measures may tap onto different cognitive or motor abilities than swaybased measures [19]; because most of the data on academic achievements and balance was time-based, it is possible that the association between balance and math is less evident using a continuous assessment of ML sway. The second explanation focus on the motor task. Specifically, in the present study dynamic balance was tested using tandem walk on a beam, which forced participants to move more slowly compared to regular walk. However, some studies have demonstrated that more active dynamic performance (e.g., running) was more likely to be associated with achievements in math rather than in language arts $[28,29]$. Similarly, it was recently reported that compared to other stances, semi-tandem dynamic balance (tandem stance with a little sideways gap between the feet) was predictive of academic achievements among 6-year-olds [30], thus strengthening the notion that task characteristics plays a role in the extent to which balance is associated with math achievements.

Following Rosenstreich, et al. [22], the current study was guided by the notion that motor performance ratio scores may tap onto the cognitive processes underlying dynamic and static balance, and thus may be more indicative of the bal- ance-academic achievements association. Indeed, when performance ratios were examined, a more complex pattern was observed. First, an interesting finding was that participants who maintained better stability on dominant foot blindfolded (ratio static 3/1), scored higher in grammar, written expression, and in total language arts score. In our view, this ratio mainly manifested participant's ability to be attentive to internal ques needed for maintaining balance in the absence of visual information, as muscle tone or information from the vestibular system [22]. Although such interoceptive attention processing has been documented in the literature (e.g., Payne, Levine, \& Crane-Godreau [31]) to the best of our knowledge it was not examined in the context of balance and academic achievements. The importance of this finding is twofold. First, understanding the role of interoceptive attention in movement may strengthen our understanding of the underlying processes of mind-body connection in general, and embodied cognition in particular. In line with this notion, it seems that similar interoceptive information processing at the fringes of consciousness [32] may also take place when attempting to maintain static balance in the absence of visual information [22] Second, because interoceptive attention may be cultivated (e.g., through mindful physical activities; Diamond \& Lee [1]), researchers and educators may employ interventions aimed at increasing interoceptive attention for promoting the cognitive abilities in the bases of academic learning [33].

More interesting, in contrast with our expectations, participants who their dynamic performance was downsized by the beanbag (ratio dynamic 2/1), scored higher in grammar, reading comprehension, and in total language arts score. Be- 
cause this ratio may be interpreted as a measure of inhibitory control over gait, two explanations may account for this effect. First, this finding may indicate that more control was associated with lower grammar grades. A possible explanation is that the acquisition of certain verbal aspects requires a reduction in control, which may also manifest in less stable motor performance. This notion is supported by a recent study which tested the association between self-regulation elements (cognitive flexibility, working memory, and inhibitory control) and academic achievements [34]. It was found that inhibitory control was linked to achievements in mathematics alone while cognitive flexibility was linked to both mathematic and verbal achievements. Hence, verbal learning may benefit from reduction in cognitive control and maintaining flexibility and openness to new information.

However, an opposite explanation may interpret high dynamic $2 / 1$ ratio as an increased control over gait, rather than a decreased control. Specifically, Dault, Frank, and Allard [35] suggested that increased cognitive load during gait task may result with increased sway frequency along with a decrease in sway amplitude, which represent more effort to monitor and adjust movement. Following this notion of tighter control over gait during the beanbag task, our findings may indicate that increased control was associated with better academic performance. However, the data collected in this study is not sufficient to prefer one explanation over the other, and further study is needed in order to better understand the association of gait beanbag task and inhibitory processes.

Altogether, our findings generally support previous findings regarding the associations between balance skills and academic performance (e.g., Planinsec; Rigoli, et al. [14,17]). However, the strength and innovation of the current study lies, in our view, in the use of performance ratios. Although studies occasionally examined dynamic balance in single compared to dual task, and static balance with eyes open compared to blindfolded (e.g., Stins, Emck, de Vries, Doop \& Beek [36]) to the best of our knowledge performance scores were scarcely employed. Such performance scores, as the difference or ratio between two conditions, are common practice in cognitive research (e.g., Rosenstreich \& Goshen-Gottstein [37]), and as demonstrated in the current study, these scores may shed more light on the associations between motor performance and other variables.

Another aspect regarding the employment of performance ratio scores is the effectiveness of cognitive assessment in young children. That is, assessing certain cognitive functions among children, as inhibitory control or attention, may be more easily carried using motor activity, especially in field studies (see also, McClelland, et al. [34]). However, one caveat in our study is that these ratios were not examined against golden standards of attention or inhibitory control (e.g., Federico, Marotta, Martella, \& Casagrande [38]), hence our interpretation of these ratios as reflecting cognitive processes should be considered with caution. One notion that stresses this caveat is that the same control condition was employed as a comparison for the beanbag gait task (dynamic task 2) and the dual-task (dynamic task 3) conditions. Because the dual-task condition consisted of increasing attentional load through visual search, and because the control condition consisted of no-load as well as no-search, the ratio between these two conditions may reflect the cost of visual rather than attentional demands. Therefore, further study is needed in order to fully understand the cognitive processes tapped by these motor performance ratios.

A second caveat in this study is the Kinect's validity in assessing motor performance. Despite its commercial success and some promising lab-based evidence [20,21], the Kinect's validity may be restricted to the assessment of rather simple gross-motor activity, and may be solely on the ML axis [39]. Nonetheless, being cost-effective, mobile, and easy to deploy, makes the Kinect a promising instrument for assessing children; hence, further study is needed in this field in order to map its potential and limitations.

Finally, a third caveat in this study was the notion that children were summoned to the school gym in groups of 6-8, with one student tested at a time while the rest watching. This was done due to time and academic constrains, but may have influenced participants' performance through collaborative inhibition [40] or through social facilitation [41].

\section{Ethics Statement}

The study was approved by the institutional ethics committee and by the school principal. Oral and written consent of participation were collected from the children and their parents, respectively. Participation was voluntary, and each student was allowed to retire at any stage of the study. Confidentiality and anonymity were kept throughout the study.

\section{Author Contribution and Conflict of Interests}

All authors contributed equally to the designing, executing, and writing of this study. The study was not funded. The authors declare no conflict of interests.

The study was not funded. The study was approved by the Peres Academic Center institutional ethical committee and by the school principle, and all participants' parents gave their written consent of participation.

\section{References}

1. Diamond A, Lee K (2011) Interventions shown to aid executive function development in children 4 to 12 years old. Science 333: 959-964.

2. Best JR, Miller PH, Naglieri JA (2011) Relations between executive function and academic achievement from ages 5 to 17 in a large, representative national sample. Learning and Individual Differences 21: 327-336.

3. Yogev Seligmann, Hausdorff JM, Giladi N (2008) The role of executive function and attention in gait. Movement Disorders 23: 329-342.

4. Cameron CE, Brock LL, Murrah WM, et al. (2012) Fine motor skills and executive function both contribute to kindergarten achievement. Child Dev 83: 1229-1244.

5. Castelli DM, Hillman CH, Buck SM, et al. (2007) Physical fitness and academic achievement in third-and fifth-grade students. J Sport Exerc Psychol 29: 239-252. 
6. Stevens TA, To Y, Stevenson SJ, et al. (2008) The importance of physical activity and physical education in the prediction of academic achievement. Journal of Sport Behavior 31: 368.

7. Bass RW, Brown DD, Laurson KR, et al. (2013) Physical fitness and academic performance in middle school students. Acta Paediatrica 102: 832-837.

8. Coe DP, Pivarnik JM, Womack CJ, et al. (2006) Effect of physical education and activity levels on academic achievement in children. Medicine \& Science in Sports \& Exercise 38: 1515-1519.

9. Coe DP, Pivarnik JM, Womack CJ, et al. (2012) Health-related fitness and academic achievement in middle school students. J Sports Med Phys Fitness 52: 654-660.

10. Shachaf M, Katz YJ, Shoval E (2013) The Unique Trio: Academic Achievement, Sport, and Gender. Education and Society 31: 17-36.

11. Nashner LM (1997) Practical biomechanics and physiology of balance. In: Jacobson GP, Newman CW, Kartush J, Handbook of balance function testing. Singular Publishing Group, San Diego (CA), 261-279.

12. Winter DA, Patla AE, Frank JS (1990) Assessment of balance control in humans. Medical Progress through Technology 16: 31-51.

13. Hrysomallis C (2011) Balance ability and athletic performance. Sports Med 41: 221-232.

14. Planinsec J (2002) Relations between the motor and cognitive dimensions of preschool girls and boys. Percept Mot Skills 94: 415-423.

15. Rizzuto T, Knight D (1993) Relations for children in grades 2, 3 , and 4 between balance skills and academic achievement. Perceptual and Motor Skills 76: 1296-1298.

16. Jansen P, Schmelter A, Kasten L, et al. (2011) Impaired mental rotation performance in overweight children. Appetite 56: 766-769.

17. Rigoli D, Piek JP, Kane R, et al. (2012) An examination of the relationship between motor coordination and executive functions in adolescents. Dev Med Child Neurol 54: 1025-1031.

18. Frick A, Möhring W (2016) A matter of balance: Motor control is related to children's spatial and proportional reasoning skills. Frontiers in Psychology 6: 2049.

19. Weiss A, Herman T, Plotnik M, et al. (2011) An instrumented timed up and go: The added value of an accelerometer for identifying fall risk in idiopathic fallers. Physiol Meas 32: 2003 2018.

20. Clark RA, Pua YH, Fortin K, et al. (2012) Validity of the Microsoft Kinect for assessment of postural control. Gait Posture 36: 372377.

21. Yang Y, Pu F, Li Y, et al. (2014) Reliability and validity of Kinect RGB-D sensor for assessing standing balance. IEEE Sensors Journal 14: 1633-1638.

22. Rosenstreich E, Levi U, Laslo-Roth R (2018) A Matter of (Inner) Balance: The Association Between Facets of Mindfulness, Attention Deficit, and Postural Stability. Mindfulness, 1-10.

23. Jensen CG, Vangkilde S, Frokjaer V, et al. (2012) Mindfulness training affects attention-or is it attentional effort? J Exp Psychol Gen 141: 106-123.
24. Faul F, Erdfelder E, Lang AG, et al. (2007) G*Power 3: A flexible statistical power analysis program for the social, behavioral, and biomedical sciences. Behav Res Methods 39: 175-191.

25. Osler CJ, Tersteeg MCA, Reynolds RF, et al. (2013) Postural threat differentially affects the feedforward and feedback components of the vestibular-evoked balance response. Eur J Neurosci 38: 3239-3247.

26. Lopes L, Santos R, Pereira B, et al. (2013) Associations between gross motor coordination and academic achievement in elementary school children. Hum Mov Sci 32: 9-20.

27. Son SH, Meisels SJ (2006) The relationship of young children's motor skills to later reading and math achievement. MerrillPalmer Quarterly 52: 755-778.

28. Donnelly JE, Lambourne K (2011) Classroom-based physical activity, cognition, and academic achievement. Prev Med 52: S36-S42.

29. Lambourne K, Hansen DM, Szabo AN, et al. (2013) Indirect and direct relations between aerobic fitness, physical activity, and academic achievement in elementary school students. Ment Health Phys Act 6: 165-171.

30. Haynes W, Waddington G, Adams RD (2018) Associations among balance, physical activity, physical fitness, and academic results in schoolchildren. Health Behavior and Policy Review 5: 57-68.

31. Payne P, Levine PA, Crane-Godreau MA (2015) Somatic experiencing: Using interoception and proprioception as core elements of trauma therapy. Front Psychol 6: 93.

32. Rosenstreich E, Ruderman L (2017) A Dual-Process Perspective on Mindfulness, Memory, and Consciousness. Mindfulness 8: 505-516.

33. Debarnot U, Sperduti M, Di Rienzo F, et al. (2014) Experts bodies, experts minds: How physical and mental training shape the brain. Front in Hum Neurosci 8: 1-17.

34. McClelland MM, Cameron CE, Duncan R, et al. (2014) Predictors of early growth in academic achievement: The Head-toes-kneesshoulders task. Frontiers in Psychology 5: 599.

35. Dault MC, Frank JS, Allard F (2001) Influence of a visuo-spatial, verbal and central executive working memory task on postural control. Gait Posture 14: 110-116.

36. Stins JF, Emck C, de Vries EM, et al. (2015) Attentional and sensory contributions to postural sway in children with autism spectrum disorder. Gait Posture 42: 199-203.

37. Rosenstreich E, Goshen-Gottstein Y (2015) Correction: Recollection-based retrieval is influenced by contextual variation at encoding but not at retrieval. PloS One 10: E0134758.

38. Federico F, Marotta A, Martella D, et al. (2017) Development in attention functions and social processing: Evidence from the Attention Network Test. Br J Dev Psychol 35: 169-185.

39. Springer S, YogevSeligmann G (2016) Validity of the kinect for gait assessment: A focused review. Sensors 16: 194.

40. Barber SJ, Rajaram S, Aron A (2010) When two is too many: Collaborative encoding impairs memory. Mem Cognit 38: 255-264.

41. Strauss B (2002) Social facilitation in motor tasks: A review of research and theory. Psychology of Sport and Exercise 3: 237-256.

DOI: $10.36959 / 447 / 343$

Copyright: (c) 2019 Shachaf M, et al. This is an open-access article distributed under the terms of the Creative Commons Attribution License, which permits unrestricted use, distribution, and reproduction in any medium, provided the original author and source are credited. 